\title{
Comunicación Masiva del Ramo Superficial del Nervio Radial con el Nervio Cutáneo Antebraquial Lateral, un Análisis Morfométrico. 1+1 $\leq 2$
}

\author{
Massive Communication Between the Superficial Branch of Radial Nerve and the Lateral \\ Cutaneous Nerve of the Forearm, a Morphometric Study. 1+1 $\leq 2$
}

"Guillermo Salgado A.; "Martin Inzunza A.; ${ }^{* *}$ Claudio Cruzat C. \& "Oscar Inzunza H.

SALGADO, A. G.; INZUNZA, A. M.; CRUZAT, C. C. \& INZUNZA, H. O. Comunicación masiva del ramo superficial del nervio radial con el nervio cutáneo antebraquial lateral, un análisis morfométrico. 1+1 <2. Int. J. Morphol., 30(3):814-820, 2012.

RESUMEN: La distribución de los ramos nerviosos sensitivos en el borde lateral y en el dorso de la mano han sido descritos con mayor exactitud en las últimas décadas, debido al avance de nuevas técnicas de diagnóstico, las cuales han permitido detectar que alrededor del $40 \%$ de la población examinada presenta algún grado de variación anatómica en el territorio de distribución de los nervios involucrados. Conocer el número de fibras que componen un ramo nervioso cutáneo de la región de la mano, ha adquirido mayor relevancia con el desarrollo de técnicas de microcirugía y de ultrasonografía, procedimientos que han demostrado la utilidad de este conocimiento en el diagnóstico y tratamiento de las lesiones nerviosas. Así, la arquitectura fascicular, el área adiposa y el área vascular de un ramo nervioso determinado constituyen datos que se ha demostrado se modifican con la edad y, en consecuencia, van condicionar la conducta terapéutica y el pronóstico de las lesiones nerviosas. En este caso presentamos una variación anatómica bilateral extremadamente rara, que involucra al ramo superficial del nervio radial y al nervio cutáneo lateral antebraquial; situación que aparece descrita en la literatura especializada sólo una vez y que modifica notablemente la inervación sensitiva del borde radial de la mano.

PALABRAS CLAVE: Nervio radial; Nervio cutáneo-lateral antebraquial; Morfometría; Fascículo; Variación anatómica.

\section{INTRODUCCIÓN}

Estudios morfológicos han demostrado que las fibras nerviosas dentro de un nervio se encuentran ordenadas en unidades estructurales bien definidas, los haces o fascículos, delimitadas por tejido conjuntivo laminar, el perineuro (Stevenson \& Lowe, 1998; Welsch, 2009). En el interior de los fascículos se disponen los axones tanto mielínicos como amielínicos.

En el último tiempo, la organización fascicular de los nervios ha comenzado a recibir mayor atención de parte de morfólogos y clínicos ya que el desarrollo de procesos diagnósticos, como la ultrasonografía, y de técnicas microquirúrgicas para la reparación de nervios han demostrado la utilidad de este conocimiento en el diagnóstico y tratamiento de las lesiones nerviosas (Marx et al., 2010a, 2010b).
En efecto, diferentes estudios señalan que la relación entre área fascicular y no fascicular varía entre diferentes nervios, incluso es diferente en distintos segmentos de un mismo nervio (Captier et al., 2005; Sladjana et al., 2008). Interesantemente, un estudio reciente señala que las fibras nerviosas agrupadas en un fascículo inervan una superficie cutánea continua y no islotes cutáneos separados (Campero et al., 2005).

Estudios morfométricos focalizados en el área no fascicular han detectado un incremento del tejido conjuntivo $\mathrm{y}$ adiposo en los nervios de los miembros superiores e inferiores de sujetos ancianos (Marx et al., 2009; Sladjana et al.). Por otro lado, Captier et al., realizando análisis morfométrico de la porción extrapetrosa del nervio facial en humanos, señalan que el número de fascículos y la canti-

\footnotetext{
" Departamento de Anatomía, Escuela de Medicina, Pontificia Universidad Católica de Chile, Santiago, Chile.

*** Escuela de Medicina, Universidad de Talca, Chile.
} 
dad de tejido conectivo se incrementa hacia la porción periférica de él. En esta misma línea, la experiencia de los cirujanos señala que la sutura exitosa de las secciones de los nervios depende la reparación temprana de la lesión, del alineamiento adecuado de los cabos nerviosos y de la edad del paciente (Dahlin, 2008).

En este caso, presentamos el análisis morfométrico de una variación anatómica bilateral extremadamente rara que involucra al ramo superficial de nervio radial (NRS), elemento que de manera íntegra se une con el ramo medial del nervio cutáneo antebraquial lateral (CABL), formando así un nervio variante (NV), nervio que da origen a los ramos SR1 y SR2 (Inzunza et al., 2011), inervando sensitivamente la región dorso-lateral de la mano; variación anatómica que hasta ahora aparecía descrita en la literatura especializada sólo una vez (Huanmanop et al., 2009).

\section{MATERIAL Y MÉTODO}

En un cadáver de sexo masculino ( 82 años de edad, donado para fines docentes, de acuerdo al libro $9^{\circ}$ del Código Sanitario (http://epi.minsal.cl/), que no presentaba lesiones en los miembros superiores, perfundido con solución fijadora vía arterial (formalina salina tamponada al 10\%) a las $28 \mathrm{hr}$ post mortem, se procedió a realizar bajo lupa $3 \mathrm{X}$ la disección bilateral del antebrazo y la mano. Se levantó la piel y se expuso el plano subcutáneo de estas regiones, siguiendo el trayecto y la convergencia entre NRS y de CABL como también el producto de esta unión, NV. Luego, en la antímera izquierda, se tomaron muestras de NRS (a nivel del tendón del músculo braquio-radial), CABL y NV (en el tejido subcutáneo); cada muestra fue seccionada transversalmente respecto del eje del nervio, dejando una porción para su inclusión en parafina y la otra fue postfijada en tetróxido de osmio e incluida en resina epóxica. De cada uno de los bloques (parafina y epon) se obtuvieron secciones de $4 \mu \mathrm{m}$ de grosor que fueron teñidas con hematoxilina eosina (H.E.) y con azul de toluidina (A.T.) respectivamente. Las secciones de los nervios fueron analizadas y fotografiadas en el microscopio de luz.
Para el análisis morfométrico se utilizó un microscopio Nikkon Labophot-2 con retículo graduado en el ocular $(1 \mathrm{~mm} / 0,001 \mathrm{~mm})$, ocupando objetivos 4x, 10x, 20x y 40x. En las secciones teñidas con H.E. se estimó directamente el área total de los nervios (NRS, CABL y NV); mientras que en las secciones tratadas con osmio y contrateñidas con A.T. se estimó el área fascicular, el área no fascicular, el área adiposa, el área vascular mediante planimetría por conteo de puntos con Sistema Test cuadrático de intervalos regulares (Mandarim-de-Lacerda, 1995). La estimación de fibras mielínicas y amielínicas de NRS, CABL y NV se realizó utilizando el método disector (Mouton, 2002).

\section{RESULTADOS}

Para cada nervio se estimó en las secciones transversales: 1.- El área total, siguiendo el epineuro. 2.- El el área fascicular total (FT), excluyendo al perineurio. 3.- El área no fascicular (NFT), que incluye el tejido conjuntivo del nervio (perineurio, tejido adiposo y vasos sanguíneos). 4.El área vascular (Vasc), midiendo el área de sección de los vasos sanguíneos del nervio. 5.- El área adiposa (Ad), considerando el tejido adiposo presente en las secciones. 6.- El número de fibras mielínicas (M) y amielínicas (Am) presentes en cada fascículo. Estos datos aparecen en la Tabla I.

El análisis morfométrico de las secciones transversales de los nervios NRS, CABL y NV nos muestra diferencias en el área y el número total de fascículos. Así, el área total de NRS es de $5,6 \mathrm{~mm}^{2}$, elemento que contiene 9 fascículos; por su parte, el área total de CABL es de $4,0 \mathrm{~mm}^{2}$ y presenta sólo 6 fascículos; para NV el área total es de 6,24 $\mathrm{mm}^{2}$, con un total de 14 fascículos. Considerando estos valores llama la atención que, en lo referido al área total de los nervios, la conexión entre NRS y CABL no representa la suma numérica de estos ramos en NV (Figs. 1, 2 y 3). Del mismo modo, en relación con el número de fascículos, la situación es similar, observándose un reordenamiento fascicular $(6+9 \neq 14)$ y también un ajuste en el área total $(5,6+4,0 \neq 6,24)$.

\begin{tabular}{lcccccccc}
\hline & \multicolumn{9}{c}{ Áreas $\left(\mathbf{m m}^{\mathbf{2}}\right)$} & \multicolumn{3}{c}{ Fibras $(\mathbf{n})$} \\
\hline Nervio & FT & NFT & Vasc & AD & Total & Mielínicas & Amielínicas & Total \\
\hline NRS & 1,1808 & 3,8 & 0,0096 & 0,08 & 5,6 & 7577,201 & 24595,928 & 32173,129 \\
CABL & 0,2576 & 3,08 & 0,136 & 0,7552 & 4 & 1430,756 & 4886,64 & 6317,396 \\
NV & 1,2308 & 4,76 & 0,088 & 0,9728 & 6,24 & 8201,298 & 22406,116 & 30607,414 \\
\hline
\end{tabular}


De los seis fascículos que presenta CABL, el menor tiene un área de $0,016 \mathrm{~mm}^{2}$ y el mayor $0,0848 \mathrm{~mm}^{2}$; para los 9 fascículos de NRS estos valores oscilan entre $0,0112 \mathrm{~mm}^{2}$ y $0,2112 \mathrm{~mm}^{2}$; para 14 fascículos de NV los números van entre $0,0032 \mathrm{~mm}^{2}$ y $0,2368 \mathrm{~mm}^{2}$. Llama la atención que NV concentra los valores extremos, es decir, el fascículo más pequeño y el más grande. Por su parte, en CABL se concentran los fascículos de menor área (Figs. 1, 2 y 3).
En los tres nervios analizados, se aprecia que el área no fascicular es considerable, representando alrededor del $70 \%$ del área total en cada uno de ellos. Por otro lado, para NRS y NV el área fascicular corresponde mas o menos al $20 \%$ del área total; sin embargo para CABL la proporción de área fascicular es del 7\%. Por su parte, el tejido adiposo es exiguo en NRS (1\%), representando alrededor del $16 \%$ en CABL y NV. El área vascular es escasa en todos los ner-

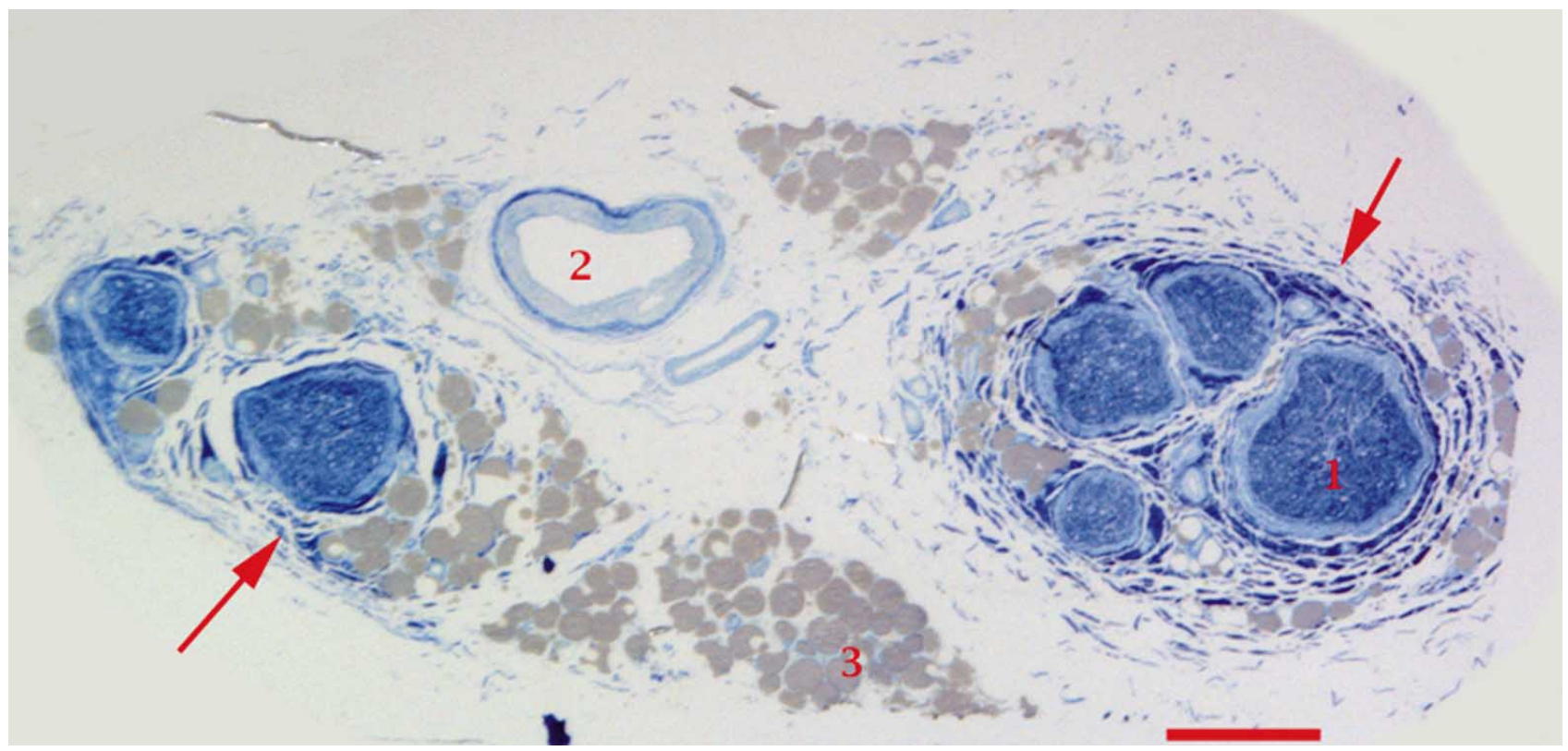

Fig. 1. Microfotografía de bajo aumento de una sección de CABL postfijada en tetróxido de osmio y contrateñida con azul de toluidina. 1= fascículo de gran tamaño. 2 = vasa nervorum. $3=$ tejido adiposo. Las flechas señala el tejido conjuntivo que envuelve a los fascículos. Una imagen similar se observa para NRS. Barra $=400 \mu \mathrm{m}$.



Fig. 2. Microfotografía de bajo aumento de una sección de NRS teñida con hematoxilina-eosina. $1=$ fascículo de gran tamaño. $2=$ vasa nervorum $3=$ tejido adiposo. Barra $=$ $500 \mu \mathrm{m}$. 


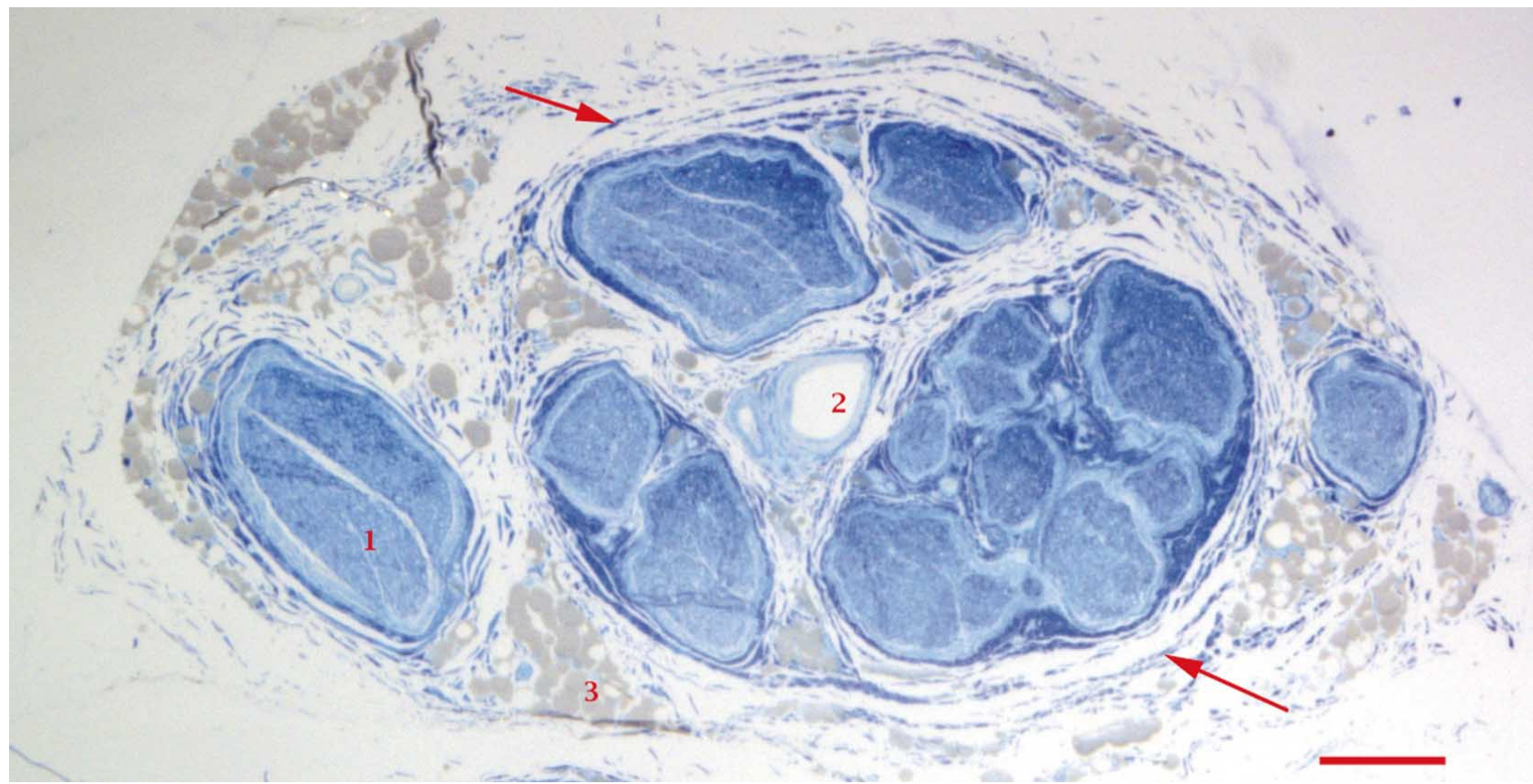

Fig. 3. Microfotografía de bajo aumento de una sección de NV postfijada en tetróxido de osmio y contrateñida con azul de toluidina. 1= fascículo de gran tamaño. 2= vasa nervorum. 3= tejido adiposo. Las flechas señala el tejido conjuntivo que envuelve a los fascículos. Barra $=300 \mu \mathrm{m}$.

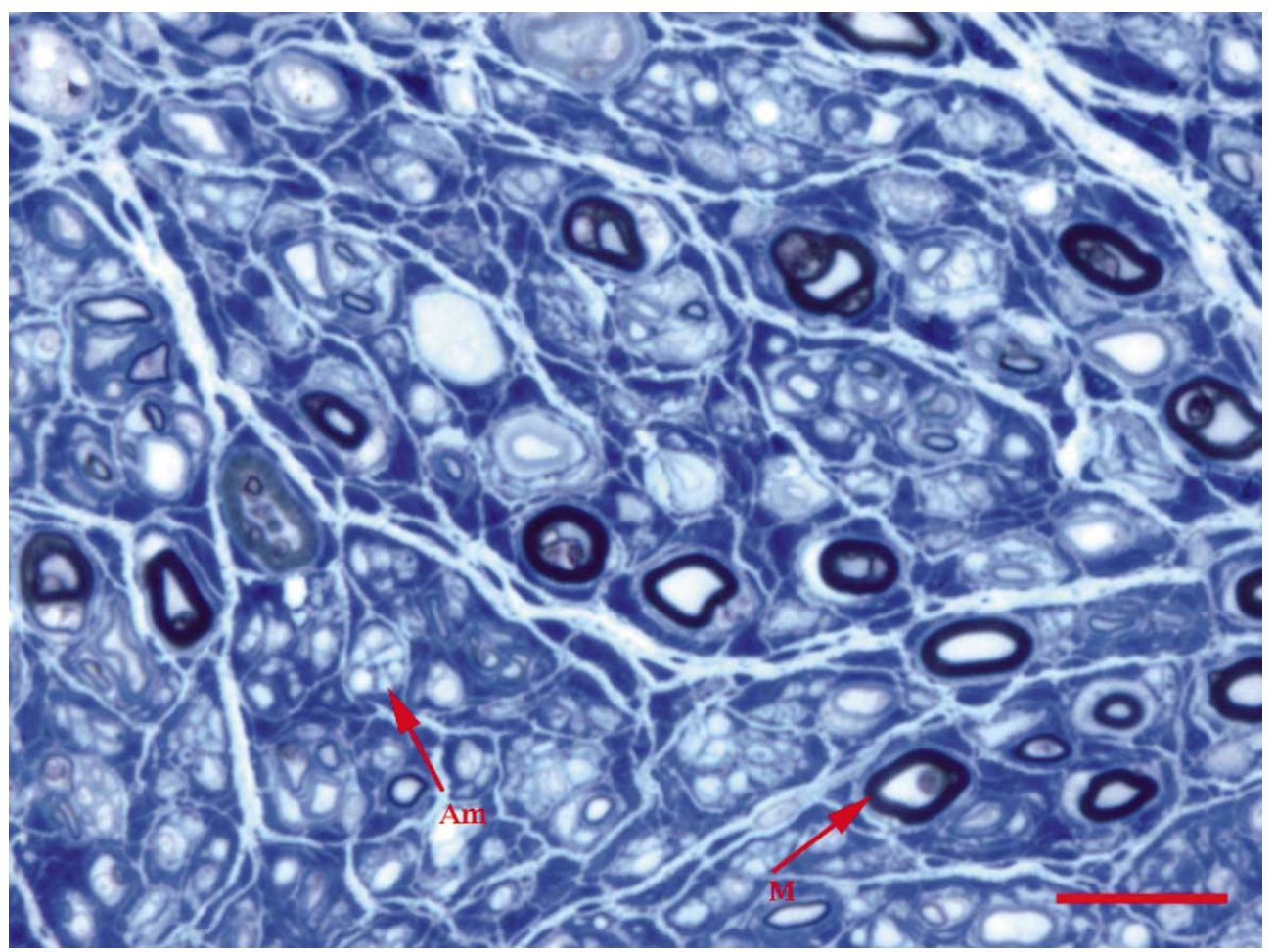

Fig. 4. Microfotografía a mayor aumento de una sección de NV postfijada en tetróxido de osmio y contrateñida con azul de toluidina. Se aprecia en esta parte del fascículo las fibras mielínicas (M), de gran tamaño, rodeadas por una gruesa vaina oscura, la mielina. En algunos nervios es posible ver el soma de la célula de Schwann (borde derecho y borde izquierdo de la imagen). También se observan numerosas y finas fibras que forman haces rodeados por una fina vaina de color azul, las fibras amielínicas (Am). Barra $=20 \mu \mathrm{m}$. vios, del orden del $2 \%$, siendo bajísima su proporción en NRS $(0,2 \%)$.

En cuanto a la calidad de las fibras nerviosas contenidas en los nervios (Fig. 4), el promedio de fibras mielínicas $\mathrm{y}$ amielínicas es el siguiente: CABL: mielínicas $=1430,756$ y amielínicas =4888,640; para NRS: mielínicas $=7577,201 \mathrm{y}$ amielínicas =24595,884; para NV: mielínicas =8201,298 y amielínicas $=22406,116$. La relación de fibras amielínicas v/s mielínicas es muy similar en todos los fascículos de los tres nervios analizados; proporción que es del orden del $75 \%$ v/s el $25 \%$ respectivamente (Tabla I). 


\section{DISCUSIÓN}

La organización fascicular de los nervios periféricos ha ido concitando progresivamente el interés de morfólogos y cirujanos ortopedistas, quienes han ponderado el valor de este conocimiento para el diagnóstico y tratamiento de las lesiones de estas estructuras neurales. Algunos territorios donde la inervación motora y sensitiva es particularmente relevante, como la cara con la porción extrapetrosa del nervio facial (Captier et al.), el miembro inferior con los nervios isquiático (Sladjana et al. y del Sol et al., 2010), sural (Jacobs \& Love, 1985), tibial y plantares (del Sol et al., 2005), fibular superficial (Vásquez et al., 2010) y plantar lateral (del Sol et al., 2006) y el miembro superior con los nervios radial (Marx et al., 2009) y CABL (Marx et al., 2010a) aparecen bien documentados.

Los estudios de la arquitectura intraneural señalan algunas características que son comunes a los nervios analizados como: 1.- El área no fascicular supera con largueza al área fascicular (Marx et al., 2009, 2010a, 2010b); situación que nosotros también detectamos en nuestro estudio (Tabla I y Figs. 1, 2 y 3). 2.- El número de fascículos y la cantidad de tejido conectivo (área no fascicular) se incrementa hacia la porción distal de los nervios (Captier et al.); hecho que parece razonable considerando las divisiones y ramos que se van emitiendo hacia la periferia. 3.- El tamaño individual de los fascículos se reduce gradualmente hacia la periferia (Marx et al., 2010b); situación perfectamente esperable considerando los ramos que se desprenden continuamente de los troncos nerviosos. 4.- En el área no fascicular el tejido adiposo se incrementa con la edad (Marx et al., 2010a); detectándose eso si una gran variabilidad individual en este punto. 5.- El área neural total se incrementa con la edad (Marx et al., 2010b); situación que parece ser consecuencia de lo señalado en el punto anterior. 6.- En la arquitectura neural no se observan diferencias entre ambas antímeras (Marx et al., 2010b); hecho que es corroborado in vivo, mediante ultrasonografía, por los mismos autores.

En nuestro estudio observamos que NRS presenta 9 fascículos, dato que coincide con lo comunicado por Folberg et al. (2009) quienes, en 20 muestras analizadas, encuentran que el número varía entre 2 y 13 ; nuestros valores también se ajustan a lo reportado por Marx et al. (2010b), quienes encuentran entre 6 y 12 fascículos. En nuestro estudio observamos para NRS un área total de $5,6 \mathrm{~mm}^{2}$ y un área fascicular total de $1,1808 \mathrm{~mm}^{2}$; por su parte, los valores que señalan Marx et al. (2010b) son de 3,6 mm² y de 1,12 $\mathrm{mm}^{2}$ respectivamente. Estas diferencias radican probablemente entonces en el área no fascicular que, como señalamos en los puntos 4 y 5 del párrafo anterior, se incrementa con la edad producto del aumento de tejido conectivo y tejido adiposo (Fig. 2).

En relación con CABL, nosotros observamos un total de 6 fascículos, un área total de $4,0 \mathrm{~mm}^{2}$, un área fascicular total de $0,2576 \mathrm{~mm}^{2}$ y un área no fascicular de $3,08 \mathrm{~mm}^{2}$. Estos datos coinciden con los reportado por Marx et al. (2010a) para ese nervio, con valores de entre 5 y 18 fascículos, un rango de área total entre $1,972 \mathrm{~mm}^{2}$ y $4,569 \mathrm{~mm}^{2}$, un rango de área fascicular total de entre $0,176 \mathrm{~mm}^{2}$ y 1,111 $\mathrm{mm}^{2}$ y un rango de área no fascicular entre $1,334 \mathrm{~mm}^{2} \mathrm{y}$ $3,887 \mathrm{~mm}^{2}$ (Fig. 1).

Por su parte NV presenta un total de 14 fascículos con área total de $6,24 \mathrm{~mm}^{2}$, un área fascicular de 1,2308 mmô y un área no fascicular de $4,76 \mathrm{~mm}^{2}$. Comparando estos valores con los de NRS y CABL, llama la atención que la convergencia total entre estos nervios, como ocurre bilateralmente en este caso, no constituya una suma numérica de los guarismos. Podemos inferir entonces que en esta convergencia nerviosa se produce un reordenamiento en la arquitectura neural; proceso que tiene como consecuencia la reducción en el número de fascículos, en el área total, en el área fascicular y en el área no fascicular. Este tipo de convergencia nerviosa no aparece descrita en la literatura especializada (Fig. 3).

NRS, CABL y, consecuentemente, NV corresponde a nervios cutáneos en cuyos fascículos se disponen sólo fibras somatosensitivas y autonómicas (Marx et al., 2009, 2010a; Campero et al.). Jacobs \& Love, realizan un pormenorizado estudio del nervio sural, nervio cutáneo de características similares a nuestro caso, y encuentran que entre los 10 y los 60 años la densidad de fibras amielínicas y mielínicas se mantiene constante, en una relación aproximada de $75 \%$ y $25 \%$, respectivamente. Después de esa edad se observa una reducción notable, del orden del $30 \%$, que afecta de manera similar a ambos tipos de fibras nerviosas, producto del desarrollo de procesos neurodegenerativos (O'Sullivan \& Swallow, 1968). Nuestros datos muestran una proporción similar entre fibras amielínicas y mielínicas y, atendiendo la edad del cadáver del cual se tomaron las muestras, la población original debió ser sin duda mayor. Paradojalmente, el área neural total aumenta con la edad (Fig. 4).

Respecto del área vascular, llama la atención que CABL y NV tienen los valores mayores debido a la presencia de vasa nervorum de calibre importante, mientras que NRS tiene vasos muy pequeños. Los nervios CALB y NV 
tienen un trayecto subcutáneo mientras que NRS emerge por debajo del tendón del músculo braquio-radial, situación topográfica que podría incidir en la disposición vascular de este nervio.

En relación con el área adiposa, los nervios de trayecto subcutáneo como CABL y NV presentan los mayores valores, siendo NRS el caso contrario. Nuevamente la situación topográfica de estos ramos nerviosos, señalada en el párrafo precedente, podría dar cuenta de esta diferencias. A este respecto es interesante consignar que la presencia de tejido adiposo dificulta y reduce las posibilidades de éxito en la reparación microquirúrgica de las lesiones nerviosas (Dumanian et al., 1999). Por otro lado, CABL es uno de los sitios donantes de elección para la obtención de injertos en la reparación microquirúrgica de nervios digitales (Tank et al., 1983) o del nervio facial (Humphrery \& Kreit, 2008). Considerando nuestros hallazgos, NRS parece ser un mejor candidato como nervio donante ya tiene una menor área adiposa.

Es interesante consignar que las muestras obtenidas de preparaciones perfundidas a las 28 horas post mortem, como en este caso, mantienen, al menos en lo que respecta a los nervios periféricos, una arquitectura microscópica adecuada para la realización de análisis histológicos y morfometría. Esta situación que maximiza el valor de ser el receptor de la voluntad postrera, donde una persona, en forma absolutamente desinteresada, regala su cuerpo para la ciencia morfológica (Inzunza, 2008).

Por último, es interesante señalar que la arquitectura fascicular de los nervios cutáneos puede ser reconocida mediante ultrasonografía (Marx et al., 2010b), detectando estos autores un alto grado de convergencia entre la visión clínica y la visión histológica.

\section{AGRADECIMIENTOS}

Los autores agradecen a la Dra. Cecilia Koenig, por su ayuda en el análisis histológico de las muestras. A la señora Mónica Pérez V., tecnóloga médica, quien proceso las muestras incluidas en epon. A la Dra. Marcia Gaete, quien facilitó equipamiento para la obtención de las microfotografías.

SALGADO, A. G.; INZUNZA, A. M.; CRUZAT, C. C. \& INZUNZA, H. O. Massive communication between the superficial branch of radial nerve and the lateral cutaneous nerve of the forearm, a morphometric study. 1+1 S2. Int. J. Morphol., 30(3):814-820, 2012.

SUMMARY: The distribution of sensory nerve branches in the lateral and the back of the hand have been described more accurately in recent decades due to advances in new diagnostic techniques, which have identified that about $40 \%$ of the population examined have some degree of anatomical variation in the distribution area of the sensitive nerves involved. The knowledge of the number of fibers forming a sensitive nerve of the hand has become more important with the development of microsurgical techniques and ultrasonography; procedures that have demonstrated the usefulness of this information in the diagnosis and treatment of nerve injuries. Thus, the fascicular architecture, adipose tissue area and the vascular area of a nerve branch, data that has been demonstrated that change with age, will determine the therapeutic and prognosis of nerve injuries. In this case we present an extremely rare and bilateral anatomical variation, involving the superficial branch of radial nerve and the lateral antebrachial cutaneous nerve, a situation that is described in the literature only once and which notably alter the sensory innervations of the radial edge of the hand.

KEY WORDS: Radial nerve; Lateral cutaneous nerve of the forearm; Fascicle; Morphometry.

\section{REFERENCIAS BIBLIOGRÁFICAS}

Campero, M.; Serra, J. \& Ochoa J. L. Peripheral projections of sensory fascicles in the human superficial radial nerve. Brain, 128(Pt 4):892-5, 2005.

Captier, G.; Canovas, F.; Bonnel, F. \& Seignarbieux, F. Organization and microscopic anatomy of the adult human facial nerve: anatomical and histological basis for surgery. Plast. Reconstr. Surg., 115(6):1457-65, 2005.

Dahlin, L. B. Nerve injuries. Curr. Orthop., 22(9):1-16, 2008.

Del Sol, M.; Olave, E.; Contreras, J. \& Vásquez, B. Morfometría de los nervios isquiático, fibular común y tibial en el Hombe. Int. J. Morphol., 28(2):385-8, 2010.

Del Sol, M.; Vasconcellos, A.; Parra, R. \& Vásquez, B. Morfometría de los nervios tibial y plantares. Int. J. Morphol., 23(4):399404, 2005.

Del Sol, M.; Vásquez, B. \& Nicklas, C. Morfometría de los ramos del nervio plantar lateral antes de su división en ramos superficial y profundo. Int. J. Morphol., 24(2):191-4, 2006.

Dumanian, G. A.; McClinton, M. A. \& Brushart, T. M. The effects 
of free fat grafts on the stiffness of rat sciatic nerve and perineural scar. J. Hand Surg. Am., 24(1):30-6, 1999.

Folberg, C. R.; Ulson, H. Jr. \& Scheidt, R .B. The superficial branch of the radial nerve: a morphologic study. Res. Bras. Ortop., 44(1):69-74, 2009.

Huanmanop, T.; Agthong, S.; Luengchawapong, K.; Sasiwongpakdee, T.; Burapasomboon, P. \& Chentanez, V. Anatomic Characteristics and Surgical Implications of the Superficial Radial Nerve. J. Med. Assoc. Thai., 90(7):1423-9, 2009.

Humphrey, C. D. \& Kriet, J. D. Nerve repair and cable grafting for facial paralysis. Facial Plast. Surg., 24(2):170-6, 2008.

Inzunza, M.; Salgado, G.; González, A.; De la Cuadra, J. C. \& Inzunza, O. Comunicación masiva del nervio radial superficial con el nervio cutáneo antebraquial lateral, implicancias anatomoclínicas. Reporte de un caso. Int. J. Morphol., 29(3):681-5, 2011.

Inzunza, O. Competencias Generales en Medicina, Rol de la Anatomía. Int. J. Morphol., 26(2):243-6, 2008.

Jacobs, J. M. \& Love, S. Qualitative and quantitative morphology of human sural nerve at different ages. Brain, 108(Pt 4):897924, 1985.

Mandarim-de-Lacerda, C. A. Métodos quantitativos em morfología. Rio de Janeiro, UERJ, 1995.

Marx, S. C.; Kumar, P.; Dhalapathy, S.; Prasad, K. \& Marx, C. A. Microanatomical and Immunohistochemical study of the human radial nerve at the antecubital fossa. Ann. Anat., 191(4):389-98, 2009.

Marx, S. C.; Kumar, P.; Dhalapathy, S.; Prasad, K. \& Marx, C. A. Microanatomical and Immunohistochemical Study of the Human Lateral Antebrachial Cutaneous Nerve of Forearm at the Antecubital Fossa and Its Clinical Implications. Clin. Anat., 23(6):693-701, 2010a.

Marx, S. C.; Kumar, P.; Dhalapathy, S.; Marx, A.; Babu, S. \& Bhat, K. Histological and ultrasonographical study of the human superficial branch of the radial nerve at distal forearm and its clinical implications. Rom. J. Morphol. Embryol., 51(4):7518, 2010b.

Mouton, P. R. Principles and practices of unbiased stereology: An introduction for bioscientists. Baltimore, The Johns Hopkins University Press, 2002.

O'Sullivan, D. J. \& Swallow, M. The fibre size and content of the radial and sural nerves. J. Neurol. Neurosurg. Psychiatry, 31(5):464-70, 1968.

Sladjana, U. Z.; Ivan, J. D. \& Bratislav, S. D. Microanatomical structure of the human sciatic nerve. Surg. Radiol. Anat., 30(8):619-26, 2008.

Stevens, A. \& Lowe, J. Histología Humana. $2^{\text {a }}$ edición. Madrid, Harcourt Brace S.A., 1998.

Tank, M. S.; Lewis, R. C. Jr. \& Coates, P. W. The lateral antebrachial cutaneous nerve as a highly suitable autograft donor for the digital nerve. J. Hand Surg. Am., 8(6):942-5, 1983.

Vásquez, B.; del Sol, M. \& Hunter, K. Morfometría de los nervios sensitivos del dorso del pie en el hombre. Int. J. Morphol., 28(4):1043-1046, 2010

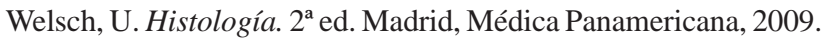

\author{
Dirección para correspondencia: \\ Dr. Guillermo Salgado Alarcón \\ Facultad de Medicina \\ Pontificia Universidad Católica de Chile \\ Santiago \\ CHILE
}

Email: gsalgado@med.puc.cl

Recibido : 12-03-2012

Aceptado: 25-06-2012 